\title{
Design and Testing of Track-Following Controllers for Dual-Stage Servo Systems with PZT Actuated Suspensions ${ }^{+}$
}

\author{
Yunfeng $\mathrm{Li}^{1}$ and Roberto Horowitz ${ }^{2}$ \\ Computer Mechanics Laboratory \\ Department of Mechanical Engineering \\ University of California, Berkeley CA 94720-1740
}

\begin{abstract}
This paper discusses the design and testing of two track-following controllers for dualstage servo systems in hard disk drives. The first controller is designed using the $\mu$ synthesis multivariable robust optimal controller design methodology. The second is designed using classical single-input-single-output (SISO) frequency shaping design techniques, based on sensitivity transfer functions decoupling of the dual-stage actuator. The controllers were implemented and tested on a disk drive with a PZT actuated suspension based dual-stage servo system. The position error signal (PES) for the servo system was obtained by measuring the slider displacement using an LDV and injecting simulated track runout. In the experiment, both designs achieved a track-mis-registration (TMR) less than $10 \mathrm{~nm}$.
\end{abstract}

Keywords: Hard disk drive, dual-stage actuator, servo control, $\mu$-synthesis

\section{Introduction}

Dual stage actuation, which combines a traditional voice coil motor (VCM) and an additional microactuator (MA) placed close to the head, has been proposed as a means of achieving higher track densities in hard disk dives (HDD), by increasing the servo bandwidth. Several design methods have been proposed for dual-stage servo design, which can be roughly categorized into two major categories. The first includes those methodologies that utilize decoupled or sequential single-input-single-output (SISO) classical frequency shaping design techniques, such as the master-slave method (Koganezawa et al., 1999), the PQ method (Schroeck et al., 1999), the parallel design method (Semba et al., 1999), and the sensitivity transfer functions decoupling or decoupled master-slave method (Mori et al., 1991; Guo et al., 1998; Li, et al., 2001), etc. The second includes those methodologies that explicitly account for coupling effects between the VCM and MA actuators and utilize multivariable optimal control design techniques such as LQG, LQG/LTR, $H_{\infty}$, or $\mu$-synthesis (Suzuki et al., 1997; Hu et al., 1999; Hernandez et al., 1999a).

\footnotetext{
+ Supported by NSF Grant 542414-55139, the National Storage Industry Consortium (NSIC) and a grant from the Computer Mechanics Laboratory (CML).

${ }^{1}$ Graduate student. Email: Yunfeng@me.berkeley.edu

${ }^{2}$ Professor and corresponding author. Email: Horowitz@me.berkeley.edu
} 
In this paper, we discuss two track-following controller designs for dual-stage servo system in HDDs. The first controller was designed using the $\mu$-synthesis toolbox (Balas et al., 1995), and is based on the design methodology presented in (Hernandez et al., 1999a). Compared with the design presented in (Hernandez et al., 1999a), the design presented in this paper was obtained using more realistic plant models and model uncertainties, and a set of weighting functions, which were determined from experimental tests. The second controller was designed using the sensitivity transfer functions decoupling method ( $\mathrm{Li}$ et al., 2001), which is similar to the design presented in (Kobayashi et al., 2001). In (Kobayashi et al., 2001), the controller was implemented using a dual-rate scheme, with the VCM loop running at a slower sampling rate than the MA loop. In this paper, both controllers were implemented with the same sampling rate, and we were able to achieve a relatively higher bandwidth.

The designed controllers were tested using a PZT actuated suspension dual-stage servo system, which utilizes PZT actuators to bend the suspension to generate a controlled fine radial head motion. The servo system's position error signal (PES) was obtained from the output of a Laser Doppler Vibrometer (LDV), which measures the absolute radial slider displacement. Since such a setup cannot directly test the servo system's track-misregistration (TMR) due to track runout, a computer generated runout signal was injected into the control system to simulate track motion.

The paper is organized as follows. Section II describes the test setup and the PZT actuated suspension and VCM models that were used to design the control systems. Section III describes the $\mu$-synthesis and sensitivity transfer functions decoupling control design methodologies, and provides a comparison of the two design results. Experimental results are presented in Section IV. Section V contains the conclusions.

\section{Test Setup and Modeling of the Dual-Stage Servo System}

\subsection{Test Setup}

Fig. 1 shows a picture of the experimental setup. A PZT actuated suspension, model Magnum-5E and manufactured by Hutchinson Technology Inc. (HTI), was mounted to an arm of the E-block of a commercial $3 \frac{1}{2}$ form factor, 5400-RPM disk drive using a small screw. In order to facilitate the installation, two other arms of the E-block were cutout. Note this is a non-standard installation and it may detrimentally affect our test results, as discussed in section 4.4.

The absolute radial displacement of the slider was measured using an LDV. The LDV has a resolution of $2.5 \mathrm{~nm}$ and the measurement gain that was used in the test is $2 \mu \mathrm{m} / \mathrm{V}$. The control circuits include a Texas Instrument TMS320C6711 DSP board, and an in-house made analog board with a 12-bits ADC, a 12-bits two channels DAC, a voltage amplifier to drive the PZT, and a current amplifier to drive the VCM.

\subsection{Dual-Stage Actuator Modeling}




\subsubsection{Modeling of the PZT Actuated Suspension}

The PZT actuated suspension frequency response was measured from the input to the voltage amplifier of the PZT actuator to the slider motion, and a transfer function was hand fitted to match this response. Fig. 2 shows the measured and simulated frequency response of the PZT actuated suspension.

The resulting transfer function of the PZT actuated suspension is

$$
G_{P Z T}=g_{P} * G_{p 1} * G_{p 2} * G_{p 3} * G_{p 4} * G_{p 5},
$$

where $g_{p}$ is the DC gain of the PZT actuator, $G_{p l}, G_{p 2}, G_{p 3}, G_{p 4}$ and $G_{p 5}$ are five major structural vibration modes. $G_{p l}$ is a sway mode

$$
G_{p 1}=\frac{\left(2 \pi \omega_{1}\right)^{2}}{s^{2}+2 s \varsigma_{1} 2 \pi \omega_{1}+\left(2 \pi \omega_{1}\right)^{2}} ; \quad \omega_{1}=8460 H z ; \quad \varsigma_{1}=0.01
$$

$G_{p 2}, G_{p 3}, G_{p 4}$ and $G_{p 5}$ are four torsional modes

$$
\begin{aligned}
G_{p i} & =\left(\frac{\omega_{i d}{ }^{2}}{\omega_{i n}{ }^{2}}\right) \frac{s^{2}+2 s \varsigma_{i n} 2 \pi \omega_{i n}+\left(2 \pi \omega_{i n}\right)^{2}}{s^{2}+2 s \varsigma_{i d} 2 \pi \omega_{i d}+\left(2 \pi \omega_{i d}\right)^{2}} ; \quad i=2,3,4,5 \\
\omega_{2 d} & =5500 H z ; \varsigma_{2 d}=0.03 ; \omega_{2 n}=5650 H z ; \varsigma_{2 n}=0.03 ; \\
\omega_{3 d} & =7300 H z ; \varsigma_{3 d}=0.03 ; \omega_{3 n}=7650 H z ; \varsigma_{3 n}=0.015 ; \\
\omega_{4 d} & =8070 H z ; \varsigma_{4 d}=0.015 ; \omega_{4 n}=8250 H z ; \varsigma_{4 n}=0.02 ; \\
\omega_{5 d} & =10650 H z ; \varsigma_{5 d}=0.01 ; \omega_{5 n}=10530 H z ; \varsigma_{5 n}=0.015 .
\end{aligned}
$$

It should be noted that the PZT actuated suspension was attached to the E-block using a non-standard installation and the slider may not have flown at the optimal flying height for this drive during the tests. These factors may have detrimentally affected the response of the PZT actuated suspension.

\subsubsection{Modeling of the VCM Actuator}

The frequency response of the voice coil motor (VCM) was measured from the input to the current amplifier of the VCM to the slider motion and the VCM model transfer function was hand fitted to match this response. Fig. 3 shows the measured and simulated frequency response of the VCM actuator.

The resulting transfer function is

$$
G_{V C M}=g_{V} * G_{V 1} * G_{V 2} * G_{V 3} * G_{V 4},
$$


where $g_{v}$ is the DC gain of the VCM, $G_{v 1}, G_{v 2}, G_{v 3}$ and $G_{v 4}$ are four major vibration modes. The transfer functions of $G_{v 1}, G_{v 2}$ and $G_{v 3}$ are

$$
\begin{aligned}
G_{V i} & =\frac{\left(2 \pi \omega_{V i}\right)^{2}}{s^{2}+2 s \varsigma_{V i}\left(2 \pi \omega_{V i}\right)+\left(2 \pi \omega_{V i}\right)^{2}} ; \quad i=1,2,3 \\
\omega_{V 1} & =135 H z ; \quad \varsigma_{V 1}=0.1 ; \\
\omega_{V 2} & =5500 H z ; \varsigma_{V 2}=0.03 ; \\
\omega_{V 3} & =8640 H z ; \varsigma_{V 3}=0.05 .
\end{aligned}
$$

$G_{v 1}$ is due to the flexibility of the printed flexible cable (PFC). $G_{v 2}$ is the butterfly mode of the VCM and E-block assembly. $G_{v 3}$ is a sway mode of the actuated suspension. $G_{v 4}$ is the same torsional mode of the PZT actuated suspension as $G_{P 3}$ as in Eq. (3).

The dash-dot line in the Fig. 3 indicates the level of additive model uncertainty used in $\mu$-synthesis design, as described in section 3.1.2.

\section{Track-Following Controller Designs}

\subsection{Track-Following Controller Design Using $\mu$-synthesis}

\subsubsection{Design Methodology}

The structured singular value, $\mu$, is a measure of how big a model perturbation must be, in order to make the control system unstable. By incorporating fictitious uncertainty blocks in the feedback system, the robust performance of the closed loop transfer function, in terms of its $H_{\infty}$ norm, can be related to the value of $\mu$. $\mu$-synthesis is a robust optimal controller design technique that attempts to minimize $\mu$ through an iterative design process. The design process described below closely follows (Hernandez et al., 1999a), (Hernandez, 1999b). The reader is referred to these references and (Balas et al., $1995)$ for more details on the $\mu$-synthesis design methodology.

In $\mu$-synthesis, model uncertainties are represented using linear fractional transformations (LFT). Disturbances and outputs must be weighted to characterize the real plant environment and the performance requirements. Fig. 4 shows the block diagram that was used to design the dual-stage track-following controller using $\mu$-synthesis.

In the block diagram, $G_{V C M}$ and $G_{M A}$ are respectively the VCM and PZT MA models. $W_{u a}, W_{u m}, \delta_{a}, \delta_{m}, \Delta_{1}$ and $\Delta_{2}$ are used to represent the model uncertainties. Details concerning the synthesis model and model uncertainties are further discussed in section 3.1.2.

Disturbance signals accounted for in the model include the track runout $r$, input disturbances to the VCM and MA, $d_{V C M}$ and $d_{M A}$, respectively, and the PES sensor noise 
$n_{P E S}$. These disturbance signals are generated by passing normalized signals $\bar{r}, \overline{d_{V C M}}$, $\overline{d_{M A}}$, and $\overline{n_{P E S}}$ respectively through weights $W_{r}, W_{d V C M}, W_{d M A}$, and $W_{n P E S}$, which can be either constants or frequency shaping filters. The weights must be selected by the designer so that actual disturbances are modeled with sufficient fidelity. The normalized signals $\bar{r}, \overline{d_{V C M}}, \overline{d_{M A}}$, and $\overline{n_{P E S}}$ each has $L 2$ norm equal to1.

The output signals in the model described by Fig. 4 are the head position error signal, $P E S$, the relative motion generated by the MA, RPES, the VCM control inputs $u_{V C M}$, and the MA control input $u_{M A}$. These signals are respectively multiplied by scaling factors, $W_{P E S}, W_{R P E S}, W_{u V C M}$, and $W_{u M A}$ to produce the weighted performance signals $\overline{P E S}$, $\overline{R P E S}, \overline{u_{V C M}}, \overline{u_{M A}}$, which are expected to have $L 2$ norm near unity. The scaling factors are selected to characterize the performance requirements.

Given a set of input and output weights and plant uncertainties, if the controller synthesized with $\mu$-synthesis achieves $\mu \leq \beta$ (ideally we want $\mu \leq 1$ ), then the closed loop transfer function, $\bar{T}$, from the normalized disturbance input

$$
\bar{d}=\left[\begin{array}{lll}
\bar{r} & \overline{d_{V C M}} & \overline{d_{M A}}
\end{array}\right]
$$

to the weighted performance signal

$$
\bar{e}=\left[\begin{array}{llll}
\overline{P E S} & \overline{R P E S} & \overline{u_{V C M}} & \overline{u_{M A}}
\end{array}\right]
$$

will have infinity norm, $\|\bar{T}\|_{\infty} \leq \beta$, for any perturbation $\Delta$ such that,

$$
\|\Delta\|_{\infty}:=\left\|\begin{array}{llll}
\delta_{a} & & & \\
& \delta_{m} & & \\
& & \Delta_{1} & \\
& & & \Delta_{2} \|_{\infty}
\end{array}\right\|_{\infty} / \beta .
$$

An interpretation of this result, in terms of RMS gains to sinusoidal inputs, which is useful for design purposes, is as follows. Assume that each element $\bar{d}_{i}$ of the disturbance input vector in Eq. (6) is a sinusoid of the form $\overline{d_{i}}=D_{i} \sin \left(\omega \cdot t+\psi_{i}\right)$ such that $\sqrt{\sum_{i=1}^{3} D_{i}^{2}} \leq 1$. Then, the steady state response of each component of the output in Eq. (7) will also be a sinusoid of the form $\overline{e_{i}}=E_{i} \sin \left(\omega \cdot t+\phi_{i}\right)$ such that $\sqrt{\sum_{i=1}^{4} E_{i}^{2}} \leq \beta$, under any set of perturbations $\delta_{a}, \delta_{m}, \Delta_{1}$ and $\Delta_{2}$, which satisfy Eq. (8). 


\subsubsection{Synthesis Model and Model Uncertainties}

From Eq. (1) - (5), the VCM and the MA transfer functions can be modeled to be the products of several individual resonance modes. The resonance frequency and damping factor of each resonance modes can have some variations. To model the parametric uncertainties in these parameters, consider the $2^{\text {nd }}$ order transfer function

$$
G=\frac{s^{2}+b_{1} s+b_{0}}{s^{2}+a_{1} s+a_{0}} .
$$

The parameters uncertainties in $a_{0}, a_{1}, b_{0}$, and $b_{1}$ can be represented using LFTs. For example, suppose there is a $\pm 10 \%$ variation in $a_{1}$, then it can be represented using the LFT

$$
a_{1}=F_{L}\left(\left[\begin{array}{cc}
a_{1} & 0.1 a_{1} \\
1 & 0
\end{array}\right], \delta_{1}\right)
$$

where $\delta_{1}$ is a real perturbation. For each torsional mode, there are 4 parameters uncertainties, and for each sway, there are 2 parameters uncertainties. We assume that the resonance frequency of each resonance mode has a $\pm 5 \%$ variation, and the damping factor has a $\pm 10 \%$ variation.

We choose the VCM model for $\mu$-synthesis design to include the first and second modes, $G_{v 1}$ and $G_{v 2}$ as defined in Eq. (5). The four parameters uncertainties in $G_{v 1}$ and $G_{v 2}$ are represented by $\Delta_{1}$

$$
\Delta_{1}=\left[\begin{array}{cccc}
\delta_{v 1} & 0 & 0 & 0 \\
0 & \delta_{v 2} & 0 & 0 \\
0 & 0 & \delta_{v 3} & 0 \\
0 & 0 & 0 & \delta_{v 4}
\end{array}\right], \quad \delta_{v i} \in R, i=1,2,3,4
$$

The unmodeled dynamics for the VCM model at higher frequency is characterized by an additive model uncertainty, $\delta_{a}$. The uncertainty weight, $W_{u a}$, was assumed to be equal to $26 \mathrm{~dB}(0.05)$, as shown by the dash-dot line in Fig. 3. The modes with magnitude in the frequency response less than $-26 \mathrm{~dB}$ are taken as uncertain and not included in the synthesis model.

The PZT MA model used in the $\mu$-synthesis design includes the sway mode and the $1^{\text {st }}$ torsional mode, $G_{p 1}$ and $G_{p 2}$ as defined in Eq. (2) and Eq. (3). We found that the inclusion of more torsional modes makes the synthesis model numerically ill conditioned and prevent a successful controller synthesis. The six parameters uncertainties in $G_{p 1}$ and $G_{p 2}$ 
are represented by $\Delta_{2}$, which is defined in a similar manner to $\Delta_{1}$ in Eq. (11) with six diagonal elements, $\delta_{m i} \in R, i=1, \ldots, 6$.

A multiplicative uncertainty, $\delta_{m}$, is used to characterize the other unmodeled modes and the gain variations of the PZT MA model. A $\pm 5 \%$ model variation is considered for all frequency by letting $W_{u m}=0.05$.

\subsubsection{Weights Selection}

The selection of the input weights $W_{r}, W_{d V C M}, W_{d M A}$, and $W_{n P E S}$ and the performance weights $W_{P E S}, W_{R P E S}, W_{u V C M}$, and $W_{u M A}$ is a very important step in the $\mu$-synthesis design methodology. Several iterations in choosing these weights may be required, in order to obtain a good final design. In our design, the performance weights $W_{P E S}, W_{R P E S}, W_{u V C M}$, and $W_{u M A}$ were chosen to be constant gains, in order to limit the magnitude of the error signals within acceptable bounds. $W_{P E S}$ was chosen to be $1 /(10 \mathrm{~nm})$, which implies that the TMR target was $10 \mathrm{~nm}$. $W_{R P E S}$ can be chosen to limit the motion of the MA to be within its allowable displacement range. However, this usually is not a problem during track following since the MA displacement range usually is sufficiently large for track following. Instead, $W_{R P E S}$ was chosen in this design to parameterize the relative displacement contribution between the VCM and the MA. Choosing $W_{R P E S}$ to be $1 /(100$ $\mathrm{nm})$ implies that the relative displacement of the MA will be required to be within 100 $\mathrm{nm}$, which in turn requires the TMR to be approximately within $100 \mathrm{~nm}$, if only the VCM actuator is utilized. $W_{u V C M}$ and $W_{u M A}$ must be chosen to bound the control input signals to within their respective saturation values. We choose $W_{u V C M}$ and $W_{u M A}$ to both be equal to $2 \mathrm{~V}$, which is the upper bound for the DAC output. It should be noted that, in general, control inputs do not reach saturation when the system is in track-following mode.

The displacement output of the LDV has a low frequency drift, which is mainly concentrated below $50 \mathrm{~Hz}$. This drift can be considered as runout to the control system. By measuring the output of the LDV after filtering it with a high pass filter, we found that the LDV and the ADC have a combined broadband measurement and quantization noise of $3.2 \mathrm{~nm}$. Thus, $W_{n P E S}$ was chosen to be $3.2 \mathrm{~nm}$.

The windage torque disturbances to the VCM and the MA were experimentally characterized by measuring the absolute open loop slider motion with the LDV. Both disturbances were assumed to be white for simplicity. The amplitudes were estimated by matching the measured power spectrum density (PSD) with the simulations, in which white excitations were fed to the VCM and the MA model respectively. The amplitude of the torque disturbance to the VCM was estimated by matching the $-40 \mathrm{~dB}$ base line of the measured PSD below $2 \mathrm{kHz}$. It was found to be equivalent to approximately $50 \mathrm{mV}$ (RMS value) acting at the current amplifier of the VCM. Thus, $W_{d V C M}$ was chosen to be $50 \mathrm{mV}$. The amplitude of the torque disturbance to the MA was estimated by matching the peak in the PSD near the sway mode frequency of the PZT actuated suspension. It was found to be equivalent to approximately $2 \mathrm{mV}$ (RMS value) acting at the voltage amplifier of the PZT MA. Thus, $W_{d M A}$ was chosen to be $2 \mathrm{mV}$. 
The runout frequency shaping weight, $W_{r}$, can be chosen to characterize actual runout as closely as possible. When combined with the PES weight $W_{P E S}$, it can also be used to shape the closed loop error rejection transfer function. If the resulting control system design achieves a peak $\mu$ value of $\beta$, then we have,

$$
\left\|W_{P E S} S W_{r}\right\|_{\infty} \leq \beta
$$

where $S$ is the closed loop sensitivity transfer function from runout $r$ to $P E S$. Define the desired closed loop sensitivity transfer function to be

$$
S_{N}=\frac{1}{W_{P E S} W_{r}}
$$

and suppose that $\beta \leq 1$. Than, the magnitude Bode plot of $S$ will be bounded below that of $S_{N}$.

In our design, $W_{r}$ was chosen to be

$$
W_{r}=\frac{0.0025(s+2 \pi \cdot 500)(s+2 \pi \cdot 2500)}{(s+2 \pi \cdot 10)(s+2 \pi \cdot 10)} .
$$

The resulting $S_{N}$ Bode plot is shown as the dash line in Fig. 5.

\subsubsection{Controller Synthesis Results}

A discrete time one-input-two-output controller was synthesized with the $\mu$-synthesis Toolbox, using the uncertainties and weights described above. The sampling frequency was chosen to be $25 \mathrm{kHz}$. A $10^{\text {th }}$ order controller was synthesized and model reduction was attempted to reduce the controller order. Tab. 1 list the peak $\mu$ values, $\beta$, for the two cases when both performance and stability are considered, and when only stability is considered, with respect to the controller order. There is almost no degradation in performance and stability robustness when the controller order is reduced from $10^{\text {th }}$ to $7^{\text {th }}$ order.

Tab. 1 Peak $\mu$ value, $\beta$, verses controller order

\begin{tabular}{|c|c|c|c|c|c|}
\hline Contr. order & $6^{\text {th }}$ & $7^{\text {th }}$ & $8^{\text {th }}$ & $9^{\text {th }}$ & $10^{\text {th }}$ \\
\hline$\beta$ (perf.+stab.) & 4.66 & 2.80 & 3.38 & 2.76 & 2.76 \\
\hline$\beta$ (only stab.) & 1.23 & 0.44 & 0.44 & 0.44 & 0.44 \\
\hline
\end{tabular}

The solid line in Fig. 5 shows the Bode plot of sensitivity transfer function $S$ of the closed loop system with the $7^{\text {th }}$ order controller, while the dash line shows the Bode plot of the desired sensitivity transfer function $S_{N}$, defined in Eq. (13). Because in our design the 
peak $\mu$ value is $\beta=2.8 \geq 1$, the magnitude Bode plot of $S$ is not totally bounded below that of $S_{N}$. By selecting a set of weights $W_{P E S}, W_{r}$ that yield a desired sensitivity transfer function $S_{N}$ with a lower bandwidth, it is possible to design a controller for which $\beta \leq 1$. However, we found during our tests that the resulting experimental result is not as good as the one presented in this paper.

The Bode plot of the open loop transfer function from $r$ to $x_{p}$ is shown as the dash line in Fig. 7. The gain cross over frequency, gain margin and phase margin are respectively $1519 \mathrm{~Hz}, 8.8 \mathrm{~dB}$ and $43.4^{\circ}$.

\subsection{Sensitivity Decoupling Track-Following Controller Design}

\subsubsection{Design Methodology}

There are several popular techniques for designing dual-stage track-following controllers, which involve some form of decoupling control and sequential designs of multiple SISO compensators. In this paper we will discuss and test the sensitivity transfer functions decoupling design method, originally proposed by (Mori et al., 1991), which is also known as the decoupled master-slave method. A block diagram for dual-stage controller design using this method is shown in Fig. 6. In Fig. 6, $r$ represents track runout. $G_{V C M}$ and $G_{M A}$ are respectively the VCM and PZT MA model transfer functions. $K_{V C M}$ and $K_{M A}$ are respectively the VCM and MA loop controllers. PES represents the motion of the head relative to the data track, while RPES represents the motion of the MA relative to the VCM. VPES represents the motion of the VCM relative to the data track. $x_{P}$ and $x_{V}$ are respectively the absolute position of the head and the VCM. For most PZT actuated suspensions, the estimate of RPES, RPES $S_{E S T}$, can be obtained by multiplying the PZT control input with the DC gain of the PZT actuator, $g_{P Z T}$, as show in Fig. 6.

The open loop transfer function from $r$ to $x_{P}$ in Fig. 6 (i.e. without the PES feedback loop being closed) is:

$$
G_{T O}=K_{V C M} G_{V C M}+K_{M A} G_{M A}+K_{M A} g_{P Z T} K_{V C M} G_{V C M} .
$$

While the closed loop sensitivity transfer function from $R$ to $P E S$ is given by

$$
S_{T}=\frac{1}{1+G_{T O}} .
$$

Assuming that RPES could be estimated perfectly, ie. $R P E S_{E S T}=R P E S$ and $g_{P Z T}=G_{M A}$, than the ideal open loop transfer function from $r$ to $x_{P}$ is:

$$
G_{T O I}=K_{V C M} G_{V C M}+K_{M A} G_{M A}+K_{M A} G_{M A} K_{V C M} G_{V C M} .
$$


and

$$
\begin{aligned}
1+G_{T O I} & =1+K_{V C M} G_{V C M}+K_{M A} G_{M A}+K_{M A} G_{M A} K_{V C M} G_{V C M} . \\
& =\left(1+K_{V C M} G_{V C M}\right)\left(1+K_{M A} G_{M A}\right)
\end{aligned}
$$

Thus, the ideal closed loop sensitivity transfer function from $r$ to $P E S$ is

$$
S_{T I}=\frac{1}{1+G_{T O I}}=\frac{1}{\left(1+K_{V C M} G_{V C M}\right)} \cdot \frac{1}{\left(1+K_{M A} G_{M A}\right)}=S_{V C M} \cdot S_{M A} \text {. }
$$

The total ideal closed loop sensitivity transfer function $S_{T I}$ of the dual-stage servo system is therefore the product of the VCM and MA loop sensitivities, respectively $S_{V C M}$ and $S_{M A}$. The dual-stage control system is stable if both the VCM loop and the MA loop are stable. Thus, the dual-stage servo control design can be decoupled into two independent controller designs: the VCM loop and the MA loop. The VCM loop controller can be designed using conventional single-stage VCM controller design techniques. The MA loop controller is designed to expand the bandwidth of the overall feedback system and provide additional error rejection.

For most PZT actuated suspensions, the low frequency response of the MA can be accurately approximated by its DC gain. Thus, $g_{P Z T}=G_{M A}+\Delta G_{M A}$, where $\Delta G_{M A}$ is small compared with $G_{M A}$ in the low frequency region (see Fig. 2 and Eq. (3)). In the high frequency region, where the resonance modes of the suspension dominate the frequency response and $\Delta G_{M A}$ is big, we can design the compensators $K_{V C M}$ and $K_{M A}$ to be small. Thus, in both cases, we have

$$
\left|K_{M A} \Delta G_{M A} K_{V C M} G_{V C M}\right|<<\left|1+K_{V C M} G_{V C M}+K_{M A} G_{M A}+K_{M A} G_{M A} K_{V C M} G_{V C M}\right|
$$

Thus an approximate decoupling can be achieved with $S_{T} \approx S_{T I}$.

\subsubsection{Controller Design Results}

The sampling frequency was chosen to be $25 \mathrm{kHz}$ for both the VCM loop and the MA loop. We choose the VCM compensator, $K_{V C M}$, to be a lead-lag controller with a notch filter to attenuate the biggest resonance peak at $5500 \mathrm{~Hz}$. The VCM loop compensator is $4^{\text {th }}$ order. The gain cross over frequency was designed to be at $700 \mathrm{~Hz}$. The resulting gain margin and phase margin are $11.7 \mathrm{~dB}$ and $50.7^{\circ}$ respectively.

The MA compensator, $K_{M A}$, was designed to be a PI controller with a notch filter to attenuate the sway mode at $8460 \mathrm{~Hz}$. The resulting compensator is $3^{\text {rd }}$ order. The gain cross over frequency was designed to be at $2000 \mathrm{~Hz}$. The resulting gain margin and phase margin are $7.9 \mathrm{~dB}$ and $66^{\circ}$ respectively. 
The VCM and MA compensators, $K_{V C M}$ and $K_{M A}$, designed above were used in the block diagram of the dual-stage control system in Fig. 6. The Bode plot of the open loop transfer function from $r$ to $x_{p}$ of the control system is shown as the solid line in Fig. 7 . The gain cross over frequency, gain margin and phase margin are respectively $1835 \mathrm{~Hz}$, $7.9 \mathrm{~dB}$ and $40^{\circ}$.

\subsection{Comparison Between the Two Designs}

\subsubsection{Frequency Responses}

Fig. 7 shows the open loop transfer function Bode plots of the two designs. As we can see from the phase plot, the $\mu$-synthesis design utilizes a phase compensation technique to maintain the stability robustness to the parameters variations in the resonance modes. The compensator places the open loop phase near the resonance frequencies, at which the gains are high, to be close to $-360^{\circ}$ and $-720^{\circ}$.

Fig. 8 shows the closed loop sensitivity transfer function Bode plots of the two designs. The decoupling design has more error rejection at low frequency, and slightly higher amplification beyond the $0 \mathrm{~dB}$ cross over frequency than the $\mu$-synthesis design. This is confirmed by the experimental results in next section.

\subsubsection{PQ Analysis}

There is a concern that destructive motion may occur when the two actuators fight each other by moving in opposite directions. A design methodology called the PQ method, which explicitly addresses this problem during controller design, was develop by Schroeck and Messner (1999). In this section, we use the PQ methodology to evaluate the relative contribution to the head position produced by the VCM and the MA with respect to frequencies, and make it sure that destructive motion does not occur. Consider the parallel block diagram in Fig. 9, and define transfer function

$$
P Q=\frac{K_{V C M}^{\prime}}{K_{M A}^{\prime}} \frac{G_{V C M}}{K_{M A}}
$$

Over the frequencies where $|P Q|_{d B}>0$, the VCM has a larger contribution to the head position, $x_{p}$; While over the frequencies where $|P Q|_{d B}<0$, the MA has a larger contribution to the head position. When $|P Q|_{d B}=0$, the magnitude of motion of the VCM and the MA is equal. Thus, to prevent destructive motion from occurring, the phase of $P Q$ must be far from $-180^{\circ}$ when the magnitude of $P Q$ is close to $0 \mathrm{~dB}$.

Fig. 10 shows the Bode plot of $P Q$ for the two designs. The $0 \mathrm{~dB}$ cross over frequency for the $\mu$-synthesis design is $760 \mathrm{~Hz}$ with a phase margin of $59.7^{\circ}$. The $0 \mathrm{~dB}$ cross over frequency for the decoupling design is $725 \mathrm{~Hz}$ with a phase margin of $78.3^{\circ}$. Thus, both designs satisfy the $P Q$ requirement. 


\subsubsection{Stability Robustness Analysis}

The stability robustness of the decoupling design is a particular concern since the decoupling is approximated by estimating RPES using the DC gain of the PZT MA. To compare and evaluate the stability robustness of the two designs, we did $\mu$-analysis using the uncertainties described in section 3.1.2 and the block diagram in Fig. 4. Notice that, in this test, only the robust stability of the controller is evaluated and the performance weights in Fig. 4 are not considered. For the $\mu$-synthesis design, the synthesized oneinput-two-output controller, $K$, was directly plugged in the block diagram to generate the stability $\mu$-plot. For the decoupling design, the controller, $K$, in Fig. 4 is

$$
K=\left[\begin{array}{c}
K_{V C M}\left(1+K_{M A} g_{P Z T}\right) \\
K_{M A}
\end{array}\right] \text {. }
$$

Fig. 11 shows the stability $\mu$-plot of the two designs, which are generated with $\mu$-tools. The peak $\mu$ value of $\mu$-synthesis design is 0.44 , which means the $\mu$-synthesis design is robust stable to the prescribed uncertainties. The peak $\mu$ value of decoupling design is 1.45, which means the it may become unstable for the worst case uncertainty perturbations described in section 3.1.2, which are actually quite conservative.

\section{Experimental Results}

\subsection{Experimental Results without Track Runout}

The two track-following control systems were implemented on the experimental setup described in section 2.1 using a Texas Instrument TMS320C6711 floating point DSP board. The controller sampling frequency was $25 \mathrm{kHz}$. Since we are using an LDV to measure the slider motion, track motion due to spindle runout and disk flutter vibrations cannot be measured. In the first test, we did not consider track runout. Thus, in this case, the role of the servo system is to overcome torque disturbances and hold the slider as still as possible. Fig. 12 shows the averaged FFT (10 averages) of the position of the slider when control is applied. The RMS value of the slider motion is $7.07 \mathrm{~nm}$ when the $\mu$ synthesis controller described in section 3.1 is utilized, while it is $6.55 \mathrm{~nm}$ when the decoupling controller described in section 3.2 is utilized.

From the FFT plots in Fig. 12, we can see that the decoupling controller has more low frequency attenuation than its $\mu$-synthesis counterpart, as predicted by the sensitivity Bode plots in Fig. 8. On the other hand, the decoupling controller also has slightly higher amplification in $2-8 \mathrm{kHz}$ frequency range than the $\mu$-synthesis controller.

\subsection{Simulated Track Runout}


In order to obtain a more realistic and complete evaluation of the dual-stage servo system performance, we used a computer generated runout signal to simulate track motion. Guo et al. (1999) measured the disk pack motion using an optical edge sensor, and found that the RMS value of disk motion is about $20 \mathrm{~nm}$ when control is not applied, and $26 \mathrm{~nm}$ when control is applied. Notice that the controller actually amplifies the disk runout because of the bandwidth limitations of the single-stage controller. Ehrlich et al. (1999) found that the TMR due to disk motion is about $30 \mathrm{~nm}$ when control is applied. The magnitude and frequency spread of the runout signal that was generated in our tests to simulate the disk motion are based on the results in (Guo et al., 1999), and (Ehrlich, 1999). Fig. 13 shows the time trace and the FFT of the simulated runout signal. Its RMS value is $20.4 \mathrm{~nm}$. The simulated runout signal was generated by passing white noise through several notch filters. Below $400 \mathrm{~Hz}$, track motion is mainly due to the spindle runout. From $400-1000 \mathrm{~Hz}$, it is mainly due to disk flutter vibrations.

\subsection{Experimental Results with Simulated Track Runout}

Fig. 14 shows the FFT of the PES recorded by the DSP when the simulated runout is injected into the control system as a reference signal. The resulting close loop PES RMS is $9.94 \mathrm{~nm}$ when the $\mu$-synthesis controller is utilized, and $9.05 \mathrm{~nm}$ when the decoupling controller is utilized.

\subsection{Discussion of the experimental results}

For a predicted linear-to-radial bit aspect ratio of 4:1, an areal density of $100 \mathrm{~Gb} / \mathrm{in}^{2}$ translates to a track density of approximately 160k tracks-per-inch (TPI), or a track pitch of $150 \mathrm{~nm}$. This implies that the TMR budget should be approximately $5 \mathrm{~nm}$ (RMS value). In our experiments, the PZT actuated suspension based dual-stage servo system achieved a PES RMS of approximately $10 \mathrm{~nm}$. However, there are several factors that may have detrimentally affected servo performance in our experimental setup, making our results too pessimistic:

First, in our experiment, two arms of the E-block are cutout. This may affected the original optimal structural dynamics of the E-block. Furthermore, the VCM may have been more susceptible to external disturbances since it becomes unbalanced. We measured the open loop slider motion, and found that its RMS was $698 \mathrm{~nm}$. In contrast, (Guo et al., 1999) reported that the open loop slider RMS motion for a similar drive was $280.8 \mathrm{~nm}$. Second, the TMR contribution from arm/suspension vibration appears to be excessive in our experiment. One possible explanation is that we mounted the PZT actuated suspension on the E-block arm using a screw, which may have introduced additional vibration modes and detrimentally affected the airflow excitation pattern. Also, the disk drive we used in the experiment was modified from an off-the-shelf single stage commercial disk drive. Thus, the E-block and the actuated suspension may not be a matched pair for optimal performance, and the slider flying height may not be set to its optimal value during the experiment. Third, the drive in our experimental setup had an opening on its cover that was used to monitor the LDV alignment. This may have also affected the airflow excitation. Finally, in our experiment, the LDV measurement noise 
was found to be $3.2 \mathrm{~nm}$, which is larger than the expected PES noise for a 160k TPI servo system.

\section{Conclusion}

Two track-following controllers were designed respectively using the $\mu$-synthesis and sensitivity transfer functions decoupling design methodologies. Both designs were experimentally validated using a dual-stage servo system with a PZT actuated suspension. The $\mu$-synthesis design has an open loop gain cross over frequency of 1519 $\mathrm{Hz}$, and a PES RMS servo precision of $9.94 \mathrm{~nm}$. The decoupling design has an open loop gain cross over frequency of $1835 \mathrm{~Hz}$, and a PES RMS servo precision of $9.05 \mathrm{~nm}$. $\mu$ analysis shows the $\mu$-synthesis design has a better stability robustness than the decoupling design.

\section{Acknowledgement}

The authors thank Robert Evans from HTI for providing us the testing samples of the PZT actuated suspension; Brian Thornton, Hany Gross and David Bogy at CML, for their help with the experimental set up; and the industrial members of the NSIC EHDR servo team, for their useful comments and feedback. 


\section{References:}

Balas, G.J., Doyle, J. C., Glover, K., Packard, A., and Smith, R. (1995), $\mu$-Analysis and Synthesis ToolBox, MUSYN Inc. and The MathWorks, Inc.

Ehrlich, R., and Curran, D. (1999), "Major HDD TMR Sources and Projected Scaling with TPI”, IEEE Trans. Magn. 35, 885-891.

Guo, L., Lee, H.S., Husson, A., and Chen, S.-H. (1999), “A Comprehensive Time Domain Simulation Tool for Hard Disk Drive TPI prediction and Mechanical/Servo Enhancemanet”, IEEE Trans. Magn. 35, 879884

Guo, W., Weerasooriya, S., Goh, T.B., Li, Q.H., Bi, C., Chang, K.T., and Low, T.S. (1998), "Dual stage actuators for high density rotating memory devices," IEEE Trans. Magn. 34, 450-455.

Hernandez, D., Park, S.-S. Horowitz, R. and Packard, A. K. (1999a), "Dual-Stage Track-Following Servo Design for Hard Disk Drive," Proc. of the 1999 American Control Conf., 4188-4121.

Hernandez, D. (1999b), Robust Servo Design for Dual-Stage Disk Drives, MS Thesis, Department of Mechanical Engineering, University of California at Berkeley.

Hu, X., Guo, W., Huang, T., and Chen, B.M. (1999), "Discrete time LQG/LTR dual-stage controller design and implementation for high track density HDDs,” Proc. of the 1999 American Control Conf., 4111-4115.

Kobayashi, M., and Horowitz, R. (2001), "Track seek control for hard drive dual-stage actuator servo systems," IEEE Trans. Magn. 37, 949-954.

Koganezawa, S., Uematsu, Y., and Yamada, T. (1999), "Dual-Stage Actuator System for Magnetic Disk Drives Using a Shear Mode Piezoelectric Microactuator,” IEEE Trans. Magn. 35, 988--992.

Li, Y., and Horowitz, R. (2001), "Mechatronics of electrostatic microactuators for computer disk drive dual-stage servo systems," IEEE/ASME Trans. Mechatronics 6, 111-121.

Mori, K., Munemoto, T., Otsuki, H., Yamaguchi, Y., and Akagi, K. (1991), “A dual-stage magnetic disk drive actuator using a piezoelectric device for a high track density," IEEE Trans. Magn. 21,5298-5300.

Schroeck, S.J., and Messner, W.C. (1999), "Dual-stage track-following servo design for hard disk drive," Proc. of the 1999 American Control Conf., 4122--4126.

Semba, T., Hirano, T., Fan, L.-S. (1999), "Dual-stage servo controller for HDD Using MEMS actuator," IEEE Trans. Magn. 35, 2271-2273.

Suzuki, T., Usui, T., Sasaki, M., Fujisawa, F., Yoshida T., and Hirai, H. (1997), "MR-48 comparison of robust track-following control systems for a dual stage hard disk drive," International Conf. on Micromechatronics for Info. and Precision Equipment. 


\section{List of Figure Captions:}

Fig. 1 A picture of the experimental setup

Fig. 2 Measured and simulated frequency responses of the PZT actuated suspension

Fig. 3 Measured and simulated frequency responses of the VCM

Fig. 4 Block diagram of the controller design using $\mu$-synthesis

Fig. 5 Closed loop sensitivity transfer function Bode plot of $\mu$-synthesis design

Fig. 6 Block diagram of the sensitivity transfer functions decoupling controller design

Fig. 7 Open loop transfer function Bode plots of the two designs

Fig. 8 Closed loop sensitivity transfer function Bode plots of the two designs

Fig. 9 A block diagram of the dual-stage system with parallel structure

Fig. 10 PQ-plot of the two designs

Fig. 11 Stability $\mu-$ plot of the two designs

Fig. 12 FFT of slider motion when control is applied

Fig. 13 Time trace and FFT of simulated track runout

Fig. 14 FFT of PES when simulated runout is injected into the control system 


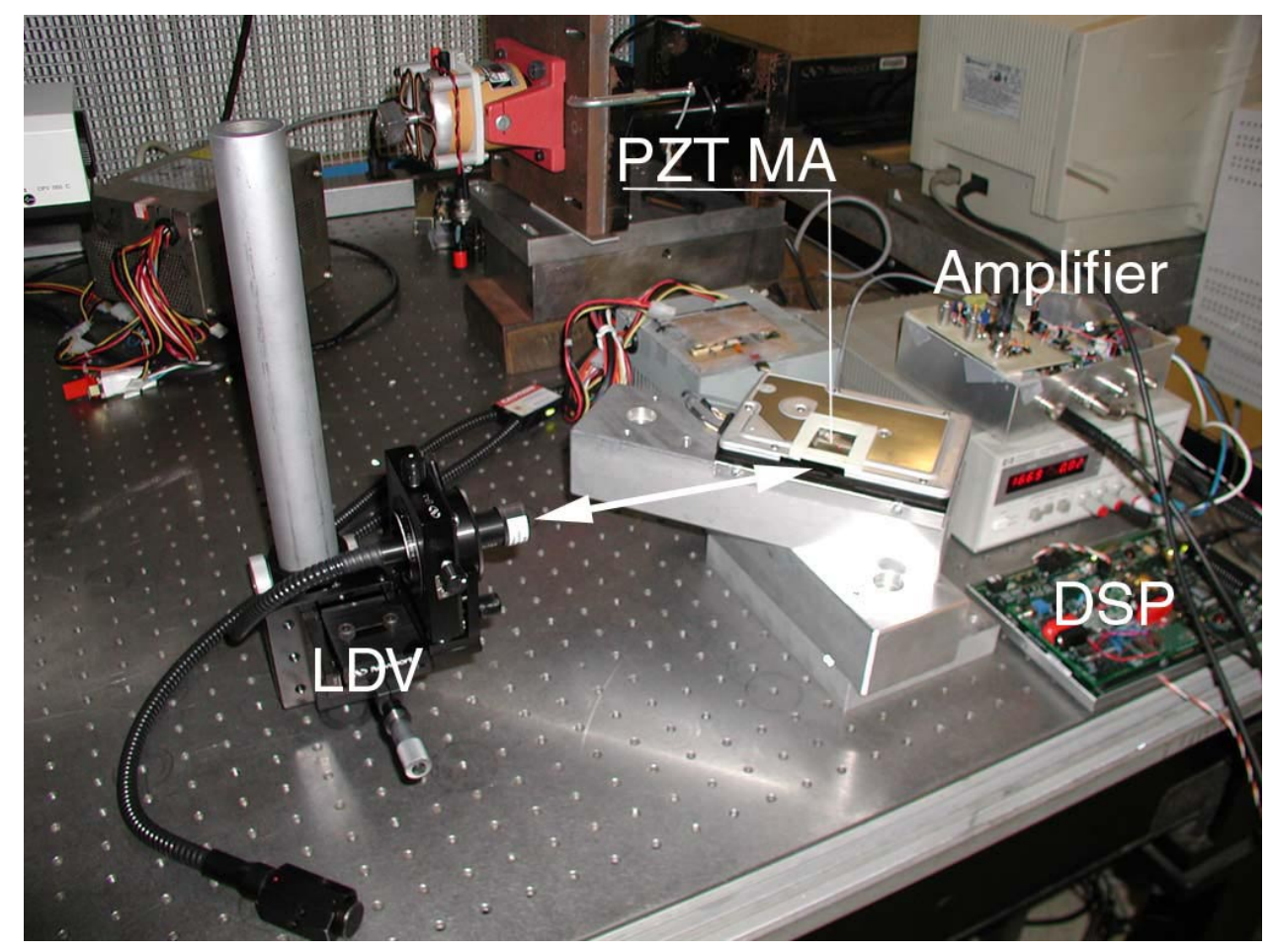

Fig. 1 A picture of the experimental setup
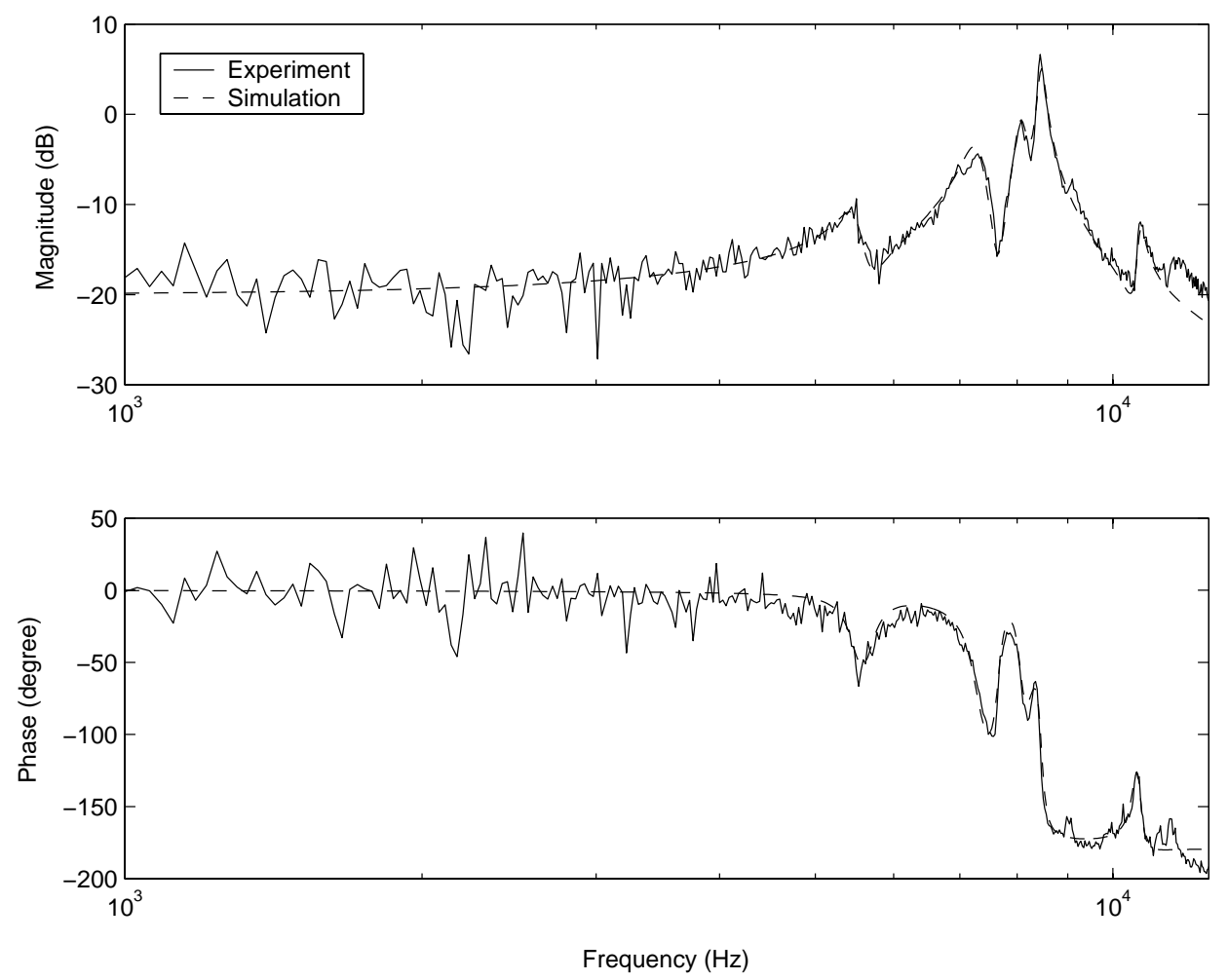

Fig. 2 Measured and simulated frequency responses of the PZT actuated suspension 

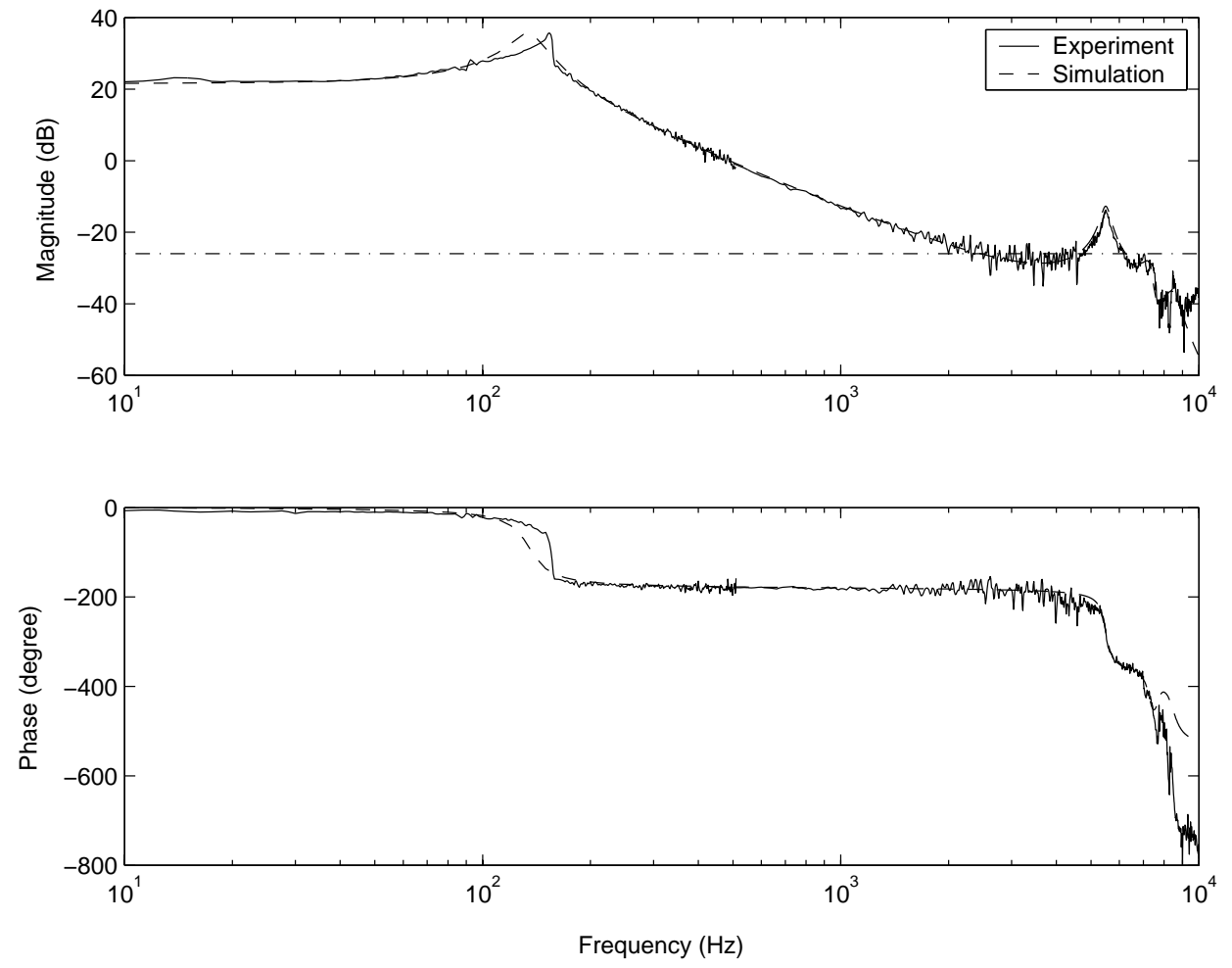

Fig. 3 Measured and simulated frequency responses of the VCM

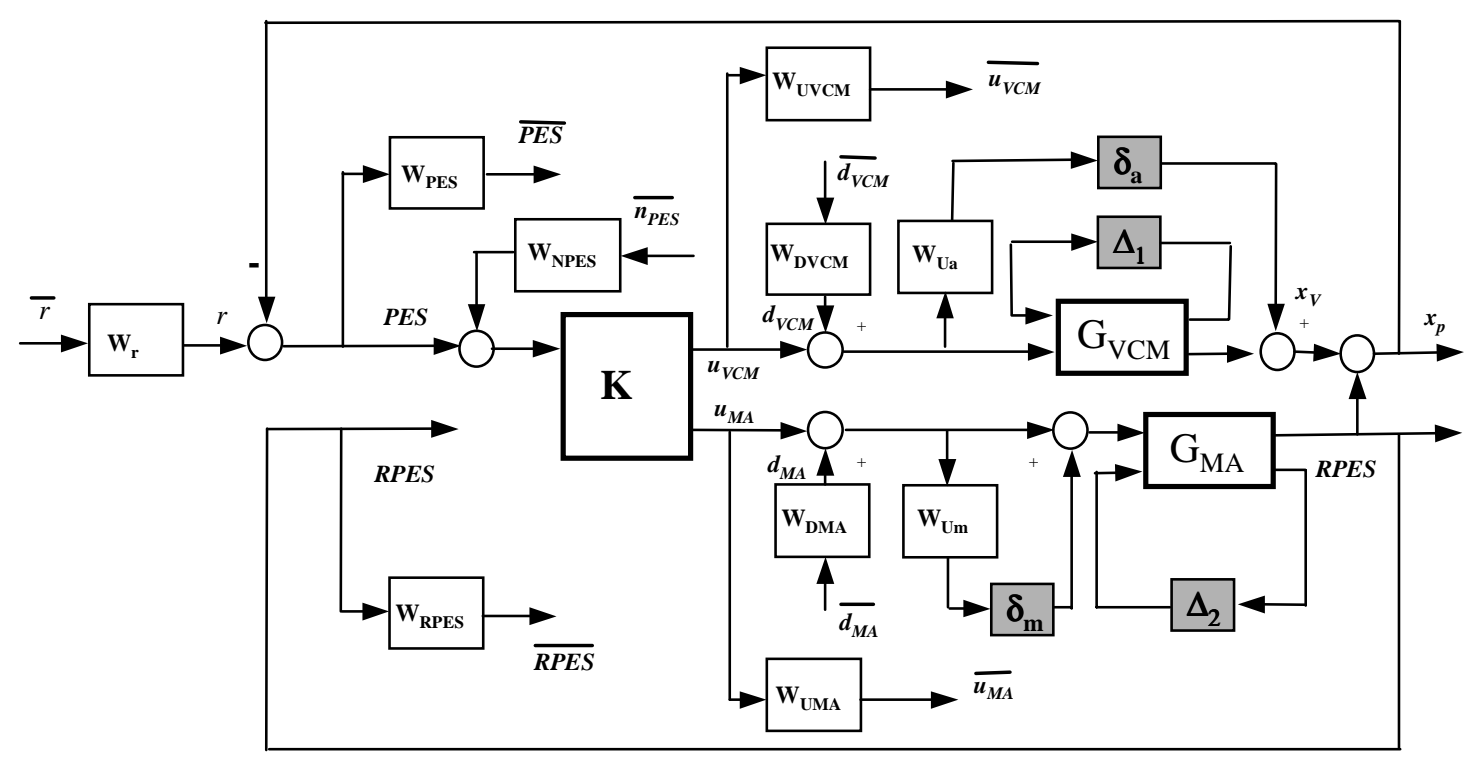

Fig. 4 Block diagram of the controller design using $\mu$-synthesis 

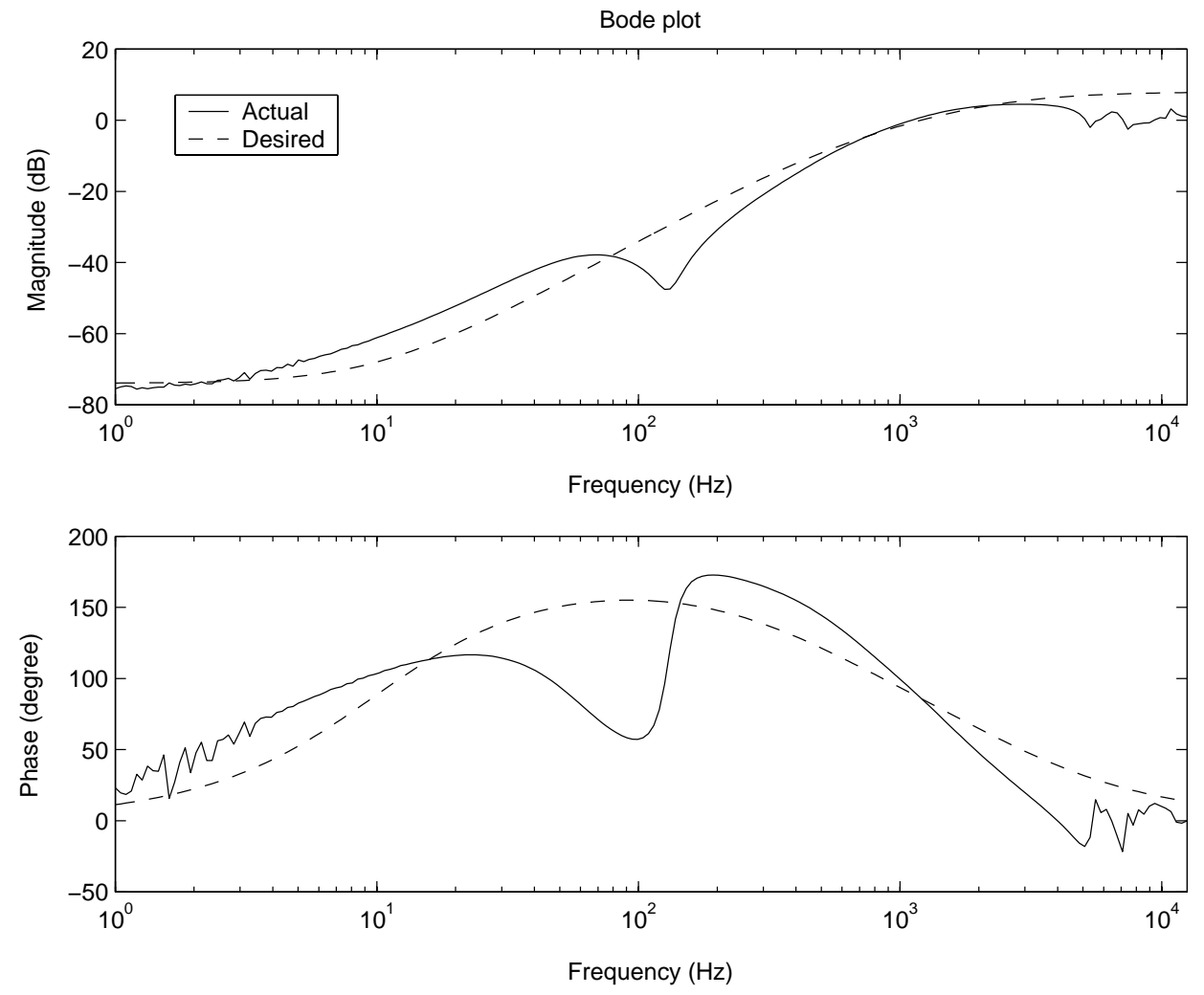

Fig. 5 Closed loop sensitivity transfer function Bode plot of $\mu$-synthesis design

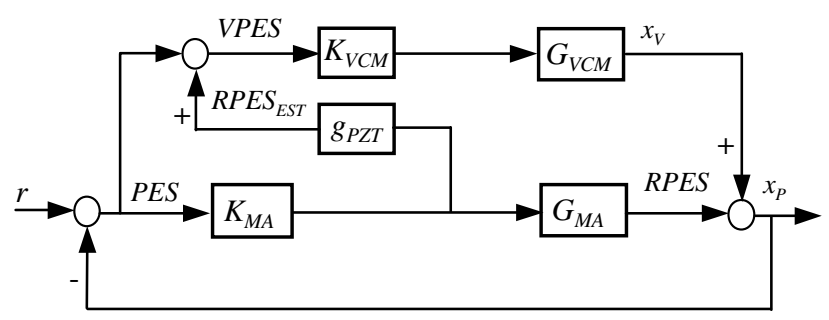

Fig. 6 Block diagram of the sensitivity transfer functions decoupling controller design 

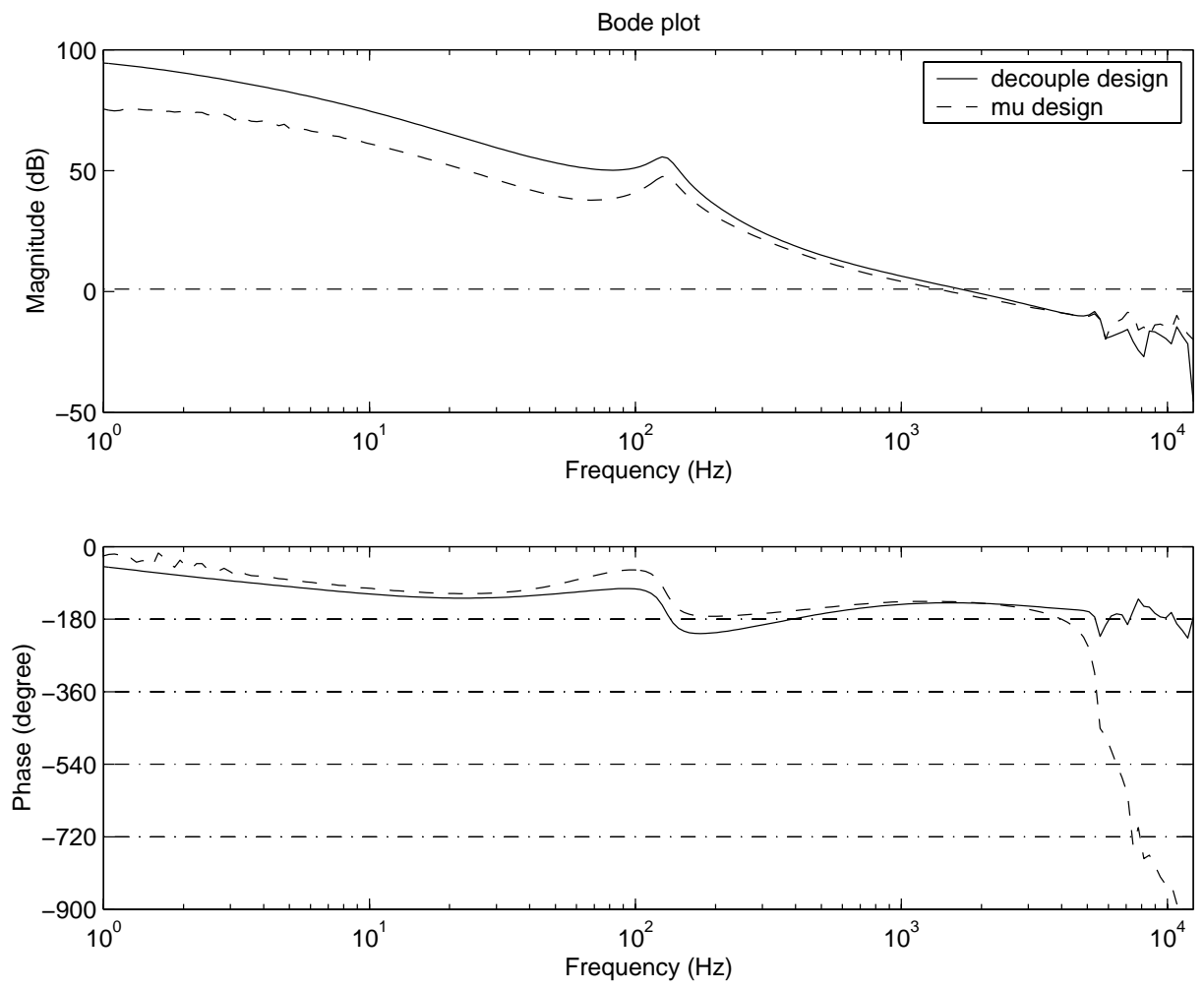

Fig. 7 Open loop transfer function Bode plots of the two designs
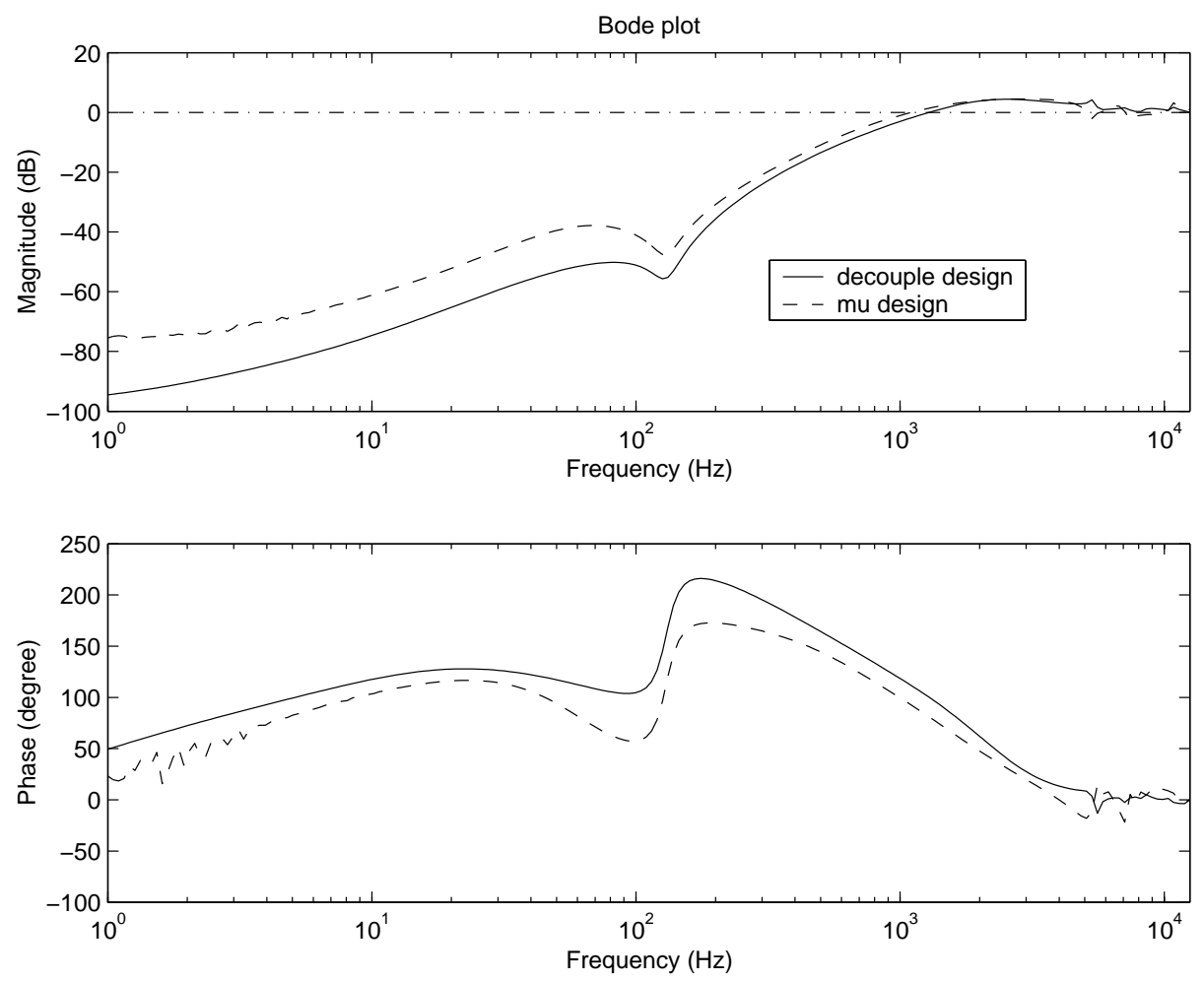

Fig. 8 Closed loop sensitivity transfer function Bode plots of the two designs 


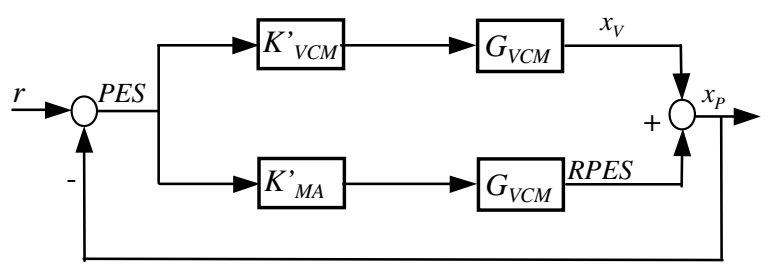

Fig. 9 A block diagram of the dual-stage system with parallel structure
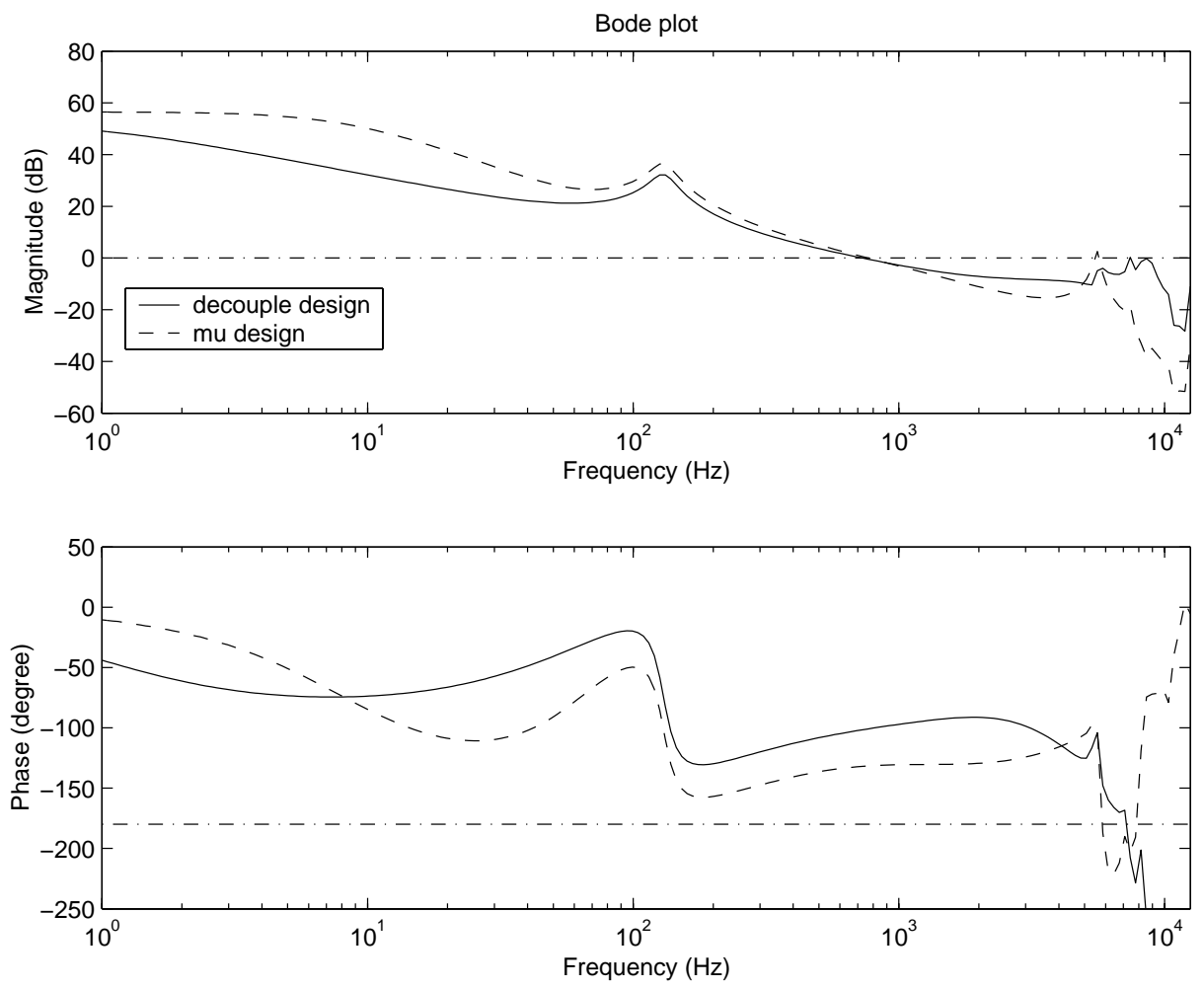

Fig. 10 PQ-plot of the two designs 


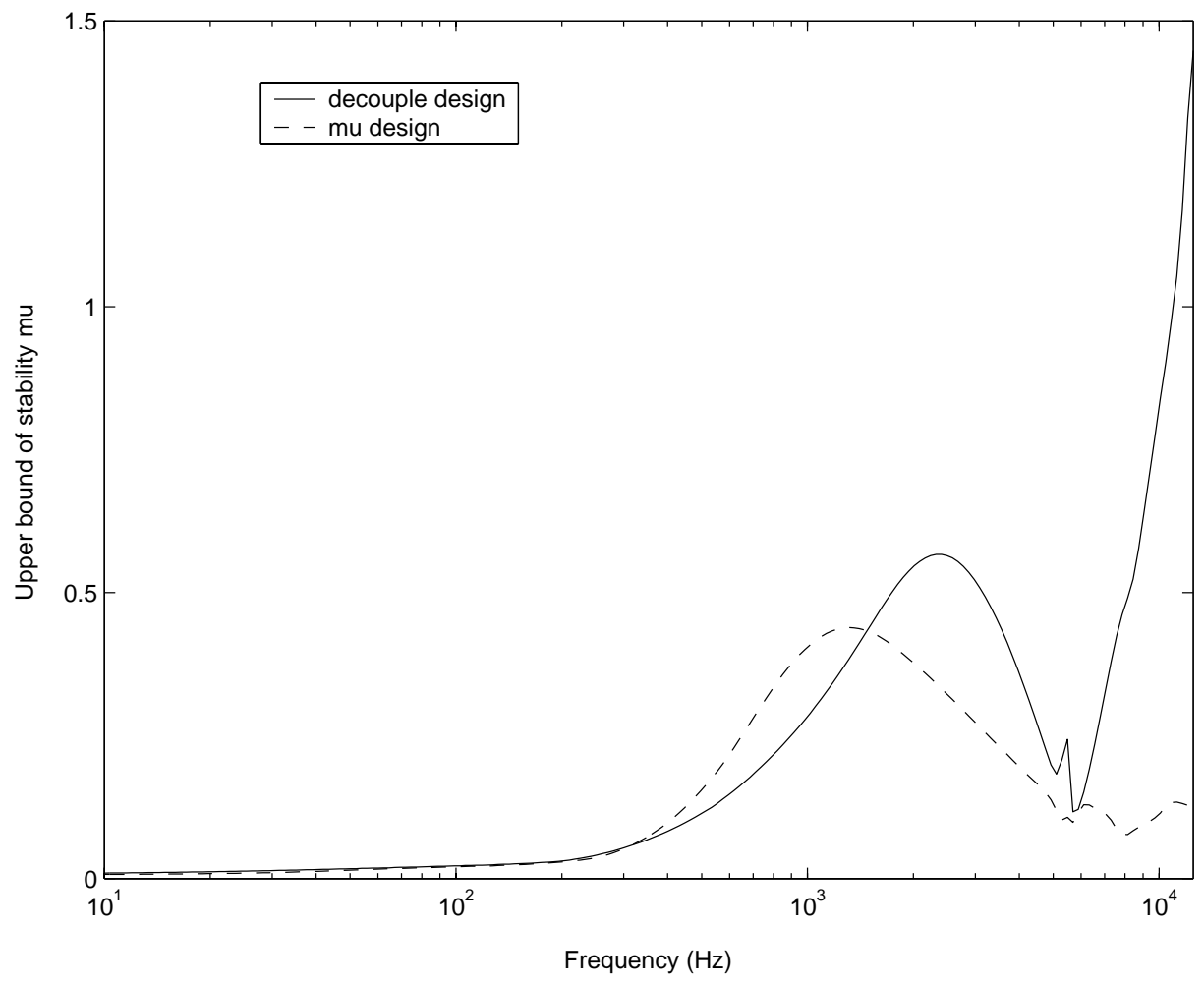

Fig. 11 Stability $\mu$-plot of the two designs
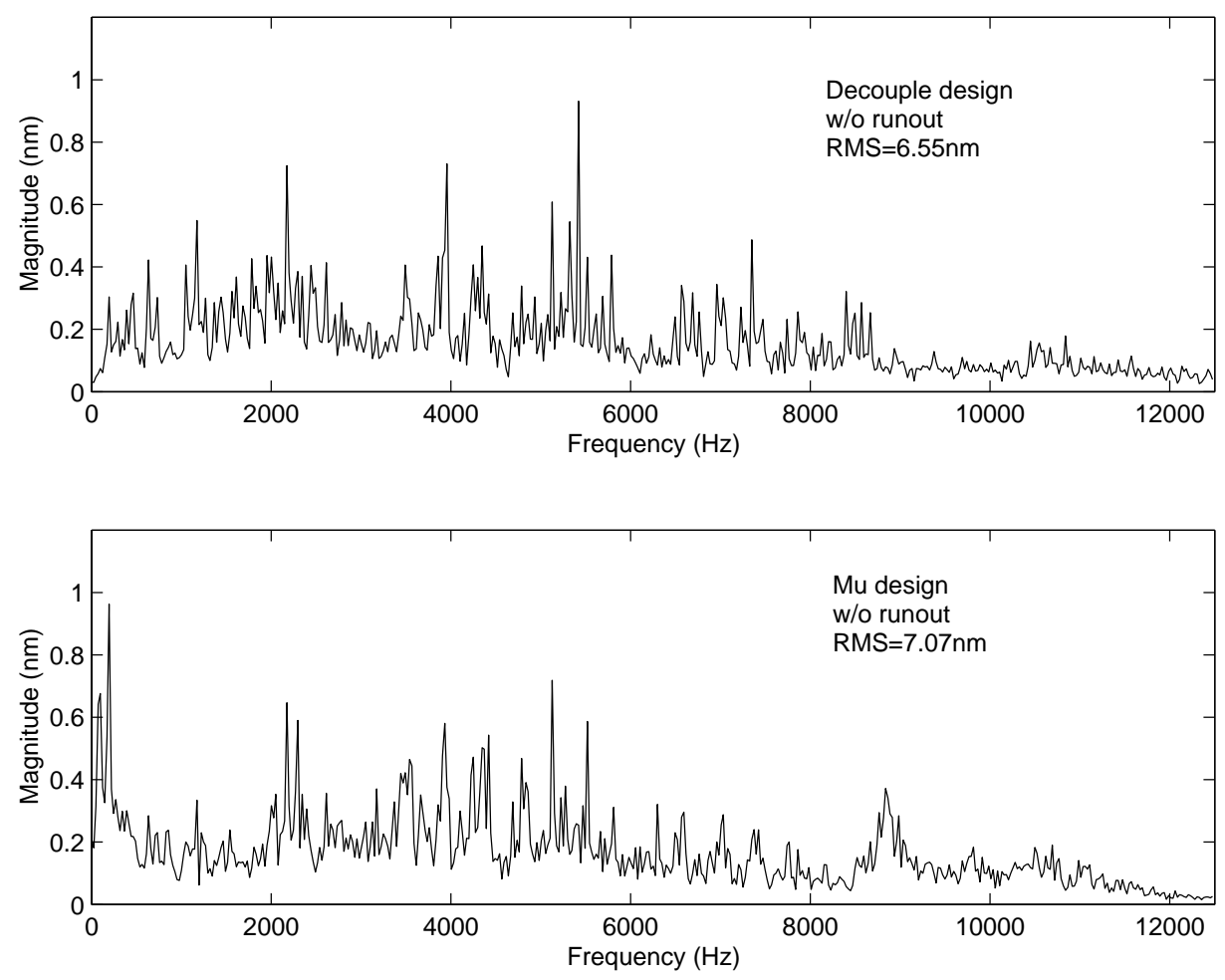

Fig. 12 FFT of slider motion when control is applied 

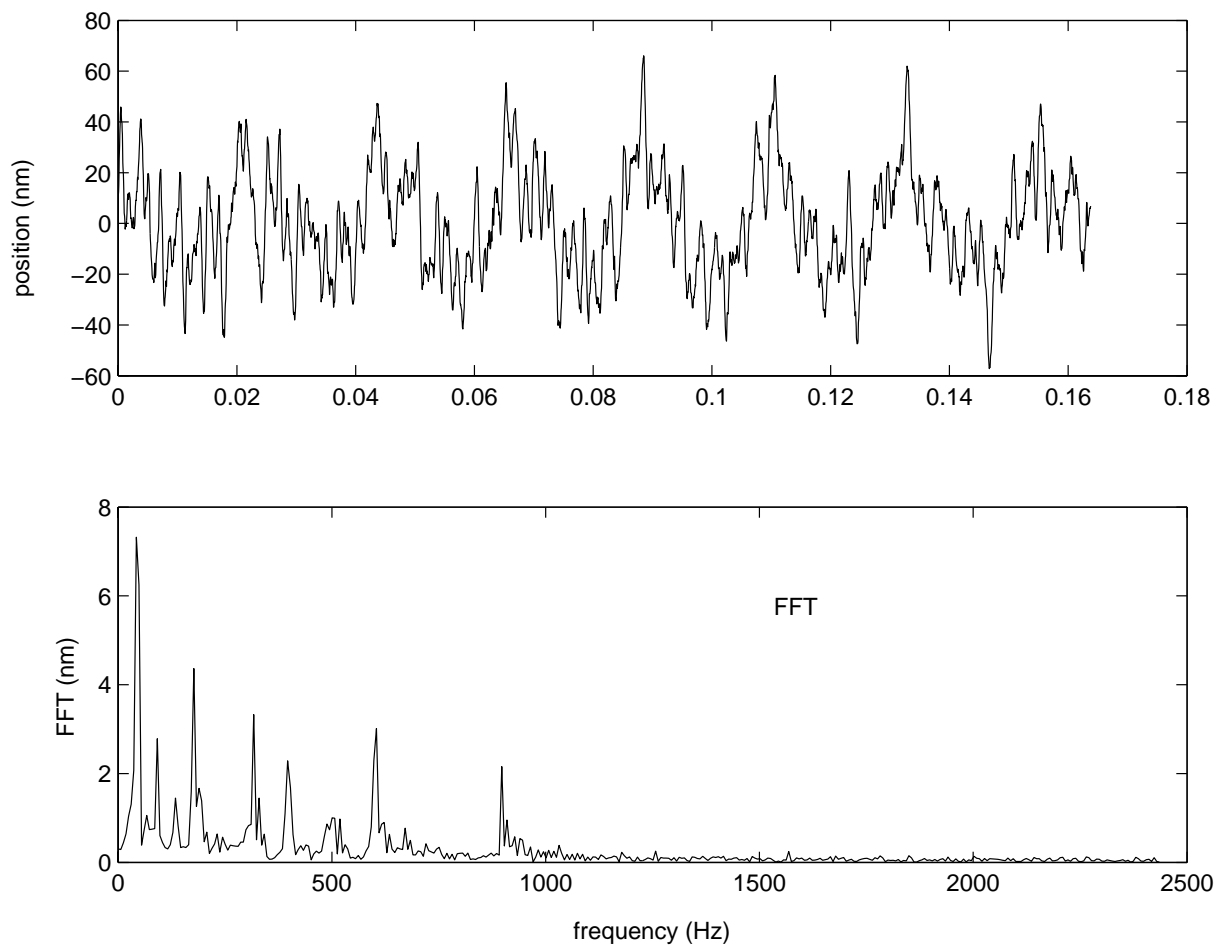

Fig. 13 Time trace and FFT of simulated track runout
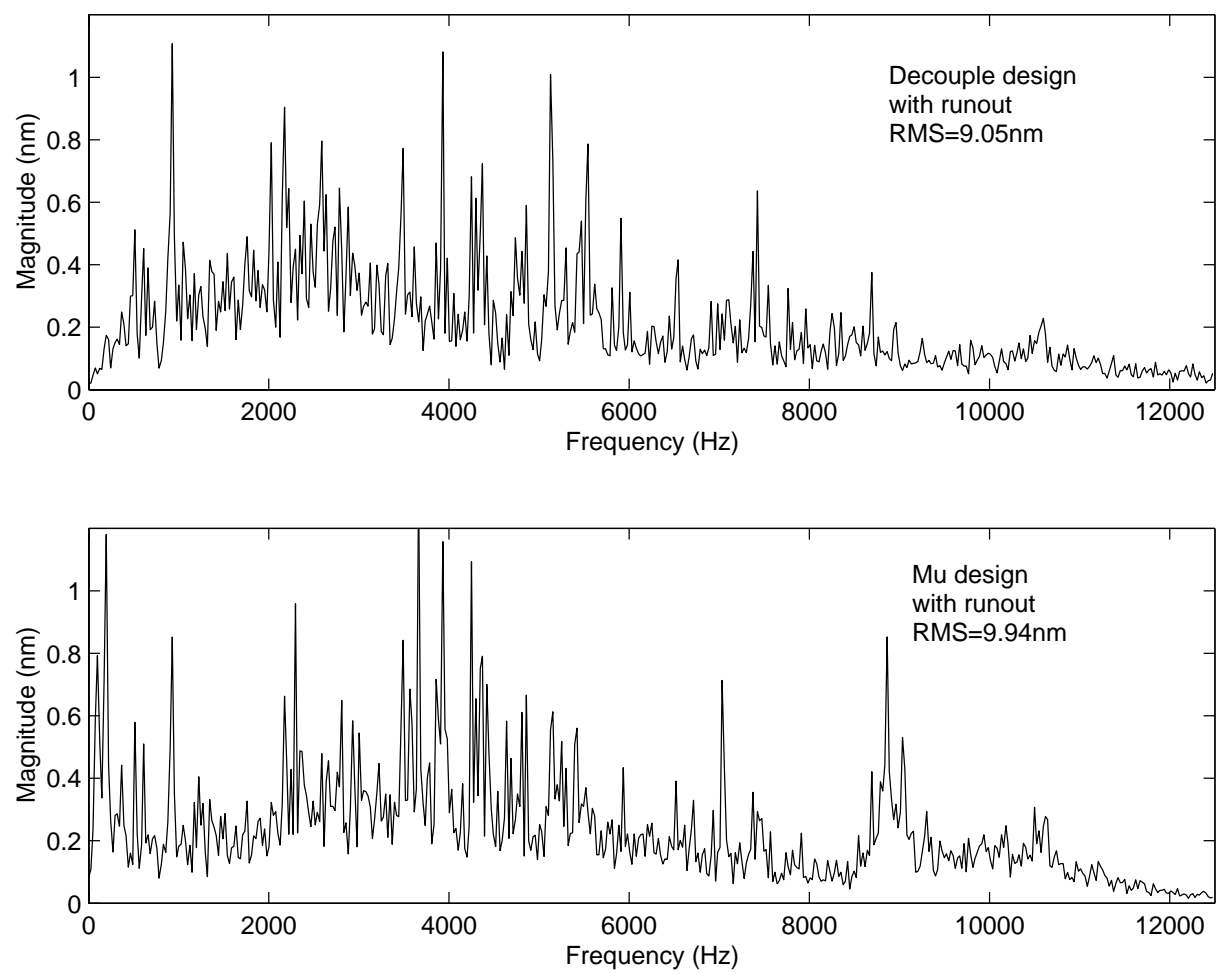

Fig. 14 FFT of PES when simulated runout is injected into the control system 